\title{
"In-bore" MRI prostate biopsy is a safe preoperative clinical tool to exclude significant prostate cancer in symptomatic patients with benign prostatic obstruction before transurethral laser enucleation
}

\author{
Angelo Porreca ${ }^{1}$, Daniele D'Agostino ${ }^{1}$, Mario Vigo ${ }^{2}$, Paolo Corsi ${ }^{1}$, Daniele Romagnoli ${ }^{1}$, Alessandro \\ Del Rosso ${ }^{1}$, Riccardo Schiavina ${ }^{3}$, Eugenio Brunocilla ${ }^{3}$, Walter Artibani ${ }^{1}$, Marco Giampaoli ${ }^{1}$ \\ ${ }^{1}$ Department of Urology, Abano Terme Hospital, Padua, Italy; \\ ${ }^{2}$ Department of Radiology, Abano Terme Hospital, Padua, Italy; \\ ${ }^{3}$ Department of Urology, University of Bologna, Bologna, Italy.
}

\begin{abstract}
Summary Introduction: Purpose of our study was to investigate the role of a negative in-bore
\end{abstract} MRI-guided biopsy (MRI-GB) in comparison to a negative multiparametric prostate MRI (mpMRI) and a contextual negative transrectal ultrasound guided biopsy of the prostate with regard to incidental prostate cancer findings in the surgical specimen of men who underwent to Holmium Laser enucleation of prostate (HoLEP) with a preoperative suspicion of prostate cancer.

Materials and methods: Data of 117 of symptomatic patients for bladder outflow obstruction who subsequently underwent to HoLEP was retrospectively analyzed form a multicentric database. All patients had a raised serum PSA and/or an abnormal digital rectal examination (DRE) with a pre-interventional mpMRI. Prostate cancer was excluded either with an en-bore MRI-GB (group "IN-BORE MRI-GB" $n=57$ ) in case of a suspect area at the mpMRI or with a standard biopsy (group "mpMRI + TRUS-GB" $n=60$ ) in case of a negative mpMRI. Preoperative characteristic surgical and histological outcomes were analyzed. Univariate and multivariate logistic regression model was performed to investigate independent predictors of incidental Prostate Cancer (iPCa).

Results: Both groups presented moderate to severe lower tract urinary symptoms: median IPSS was 19 (IQR: 17.0-22.0) in the IN-BORE MRI-GB group and 20 (IQR: 17.5-22.0) in the $m p M R I+T R U S-G B(p=0.71)$. No statistically significant difference was found between the two groups besides total prostate volume with $68 \mathrm{cc}$ (IQR: 58.0-97.0) in the IN-BORE MRI-GB group and $84 c c$ (IQR: 70.0-115.0) in the mpMRI + TRU-GB group ( $p=0.01)$ No differences were registered in surgical time, removed tissue, catheterization time, hospital stay and complications rate. No different rates $(p=0.50)$ of iPC a were found in the IN-BORE MRI-GB group (14\%) in comparison with mpMRI + TRUS-GB group (10\%); pT stage and ISUP Grade Group in iPCa stratification were comparable between the two groups. In multivariate analysis a statistically significant correlation with age as an independent predictive factor of iPCa was found (OR 1.14; 95\% CI: 1.02-1.27; $p=$ 0.02 ) while no correlations were revealed with PSA (OR 1.12; 95\% CI: 0.99-1.28; $p=0.08$ ) and a negative in-bore MRI-GB (OR 1.72; 95\% CI: 0.51-5.77; $p=0.37$ ).

Conclusions: Including a mpMRI and an eventual in-bore MRIGB represents a novel clinical approach before surgery in patients with symptomatic obstruction with a concomitant sus- picion of PCa, leading to low rate of iPCa and avoiding unnecessary standard TRUS-GB biopsies.

KEY WORDS: Magnetic resonance imaging; Holmium laser enucleation of the prostate; Prostate biopsy; Prostatic enlargement; Prostate cancer.

Submitted 29 May 2019; Accepted 2 August 2019

\section{INTRODUCTION}

One of the most common non-malignant disease in aging men is represented by benign prostate enlargement (BPE) (1) which might drives to bladder outlet obstruction (BOO) with consequent affected quality of life (QoL) leading to the necessity of a surgical procedure.

During the preoperative work-up, a prostate cancer (PCa) diagnosis might be arise and whenever its presence is suspected, its exclusion is necessary since prostate cancer might represent an heavy burden in quality of life (2) and both an accurate diagnosis and risk stratification are mandatory for an adequate disease management $(3,4)$. In men with a raised serum prostate specific antigen (PSA) and/or abnormal digital rectal examination (DRE) the standard of care in order to rule out PCa is represented by a 10-12 core ultrasound guided transrectal biopsy of the prostate (TRUS-GB) (5) which several times leads to either false negative results or non-clinically significant PCa $(6,7)$

During the last years, several new imaging techniques such as magnetic resonance imaging (MRI) (8) and positron emission tomography (PET) (9), were introduced in the clinical practice in order to diagnose and stage PCa. A multiparametric Magnetic Resonance Imaging (mpMRI) of the prostate combines both functional and morphological studies and demonstrated to be a valuable tool for PCa diagnosis with high sensitivity and specificity (10). Performing a targeted biopsy to mpMRI suspect areas might reduce the numbers of necessary biopsies and lower the non-clinically significant PCA rates (11). Several targeting techniques were proposed: visual estimation TRUS-GB (cognitive technique), software co-reg- 
istered MRI-ultrasound fusion (fusion technique) and inbore MRI-guided biopsy (MRI-GB). In-bore MRI-GB has the advantage to provide the greatest probability to sample suspected areas since is performed with a direct and real-time proof of the correct sampling (12) especially in case of high volume prostate.

Holmium laser enucleation of the prostate (HoLEP) represent an endoscopic surgical technique that allows to obtain patients' symptoms relief. HoLEP is reported to be applicable to all prostate sizes and to represent a safe, efficient and time-durable surgical solution to patients (13).

Differently to other laser techniques for the treatment of symptomatic BPE, HoLEP is able to retrieve and adequate enucleated prostatic adenoma tissue, better than transurethral resection of the prostate (TURP) and comparable to open simple prostatectomy (14). Purpose of our study was to investigate the role of a negative in-bore MRIGB in comparison to a negative mpMRI and a contextual negative transrectal ultrasound guided biopsy of the prostate with regard to incidental prostate cancer findings in the surgical specimen of men who underwent to HoLEP with a preoperative suspicion of prostate cancer due to a raised PSA and/or and abnormal DRE.

\section{Patients AND methods}

\section{Population and study design}

Data was retrospectively retrieved from a multicentric database of patients affected by symptomatic BOO due to BPE who underwent to HoLEP from January 2017 to December 2018.

Surgical indication was given in case of persistent bladder outflow obstruction symptoms, International Prostatic Symptoms Score (IPSS) higher than 8, peak urinary flow $\left(\mathrm{Q}_{\max }\right) \leq 15 \mathrm{ml} / \mathrm{s}$, non-responsiveness to medical therapies (alfa blockers and/or 5 $\alpha$-reductase inhibitors 5ARI), acute and chronic urinary retention or renal function impairment due to BOO. All patients selected from the database had exclusively a pre-operative suspicion of $\mathrm{PCa}$ (total PSA > $4 \mathrm{ng} / \mathrm{mL}$ and /or abnormal DRE) and underwent to a pre-interventional prostate mpMRI.

Multiparametric MRI was performed with a $1.5 \mathrm{~T}$ whole body scanner (Achieva XR; Philips Medical Systems, Best, Netherlands) with a 32-channels phased-array surface and without an endorectal coil. Morphological studies consisted in Turbo Spin Echo (TSE) T2-weighted sequences in sagittal, axial and coronal planes while functional studies were obtained through Diffusion Weighted Imaging (DWI) and Dynamic Contrast Enhanced-MRI (DCE-MRI). MpMRIs were exclusively conducted in one of the two involved centers and images were evaluated by two high experienced UroRadiologists according to PI-RADSv2, based on ESUR guidelines for the evaluation and reporting of prostate mpMRI (15).
Patients with a suspicious area at the mpMRI (PI-RADS v2 Score $\geq 3$ ) underwent to in-bore MRI-GB (2 cores taken per suspect lesion), resulted negative for $\mathrm{PCa}$ and then were scheduled for HoLEP. Individuals with a negative mpMRI (PI-RADS -v2 Score < 3) underwent to an additional standard random TRUS-GB (10 or 12 cores were taken based on the prostate volume), resulted negative for PCa and then scheduled for HoLEP.

Pre-operative collected data included age, total PSA, DRE, prostate and adenoma volume either at mPMRI or at transrectal ultrasound, PSA density, $\mathrm{Q}_{\max }$, IPSS, QoL, post-voided residual volume (PRV), drug assumption, previous acute urinary retention. The following peri- and post-operative parameters were evaluated: surgical time, removed tissue weight, catheterization time, hospital stay, peri-operative complications, presence of incidental Prostate Cancer (iPCa), pT stage and International Society of Urological Pathology (ISUP) Grade Group of each iPCa.

\section{Conduct of the in-bore MRI-guided biopsy (MRI-GB)}

Biopsies were performed by two urologists with consolidated experience in MRI-GB. Oral antibiotic prophylaxis was started one day before the procedure prolonged for at least 2 days. Peri-prostatic nerve blockade local anesthesia with lidocaine $2 \%$ was executed for patients' pain relief. Biopsies were performed transrectally, with patients in a

\section{Figure 1.}

The non-magnetic MR-compatible biopsy device fixed on the table top of the magnet.

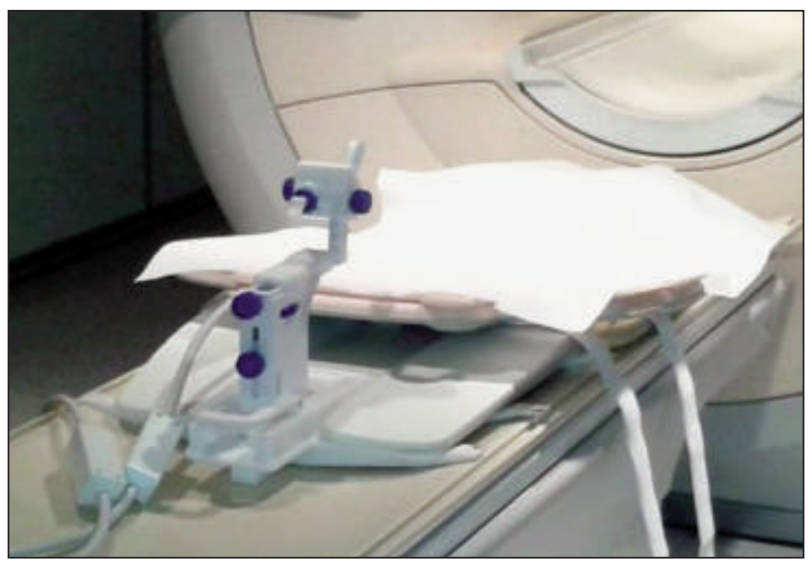

Figure 2.

The Gadolinium-filled needle guide properly identified in a sagittal T2-weighted image (A); the dedicated software (DynaCAD, Invivo, Gainesville, FL) shows the $3 D$ adjustments through automatic calculation enabling the proper calibration of the biopsy needle to the target lesion (B).

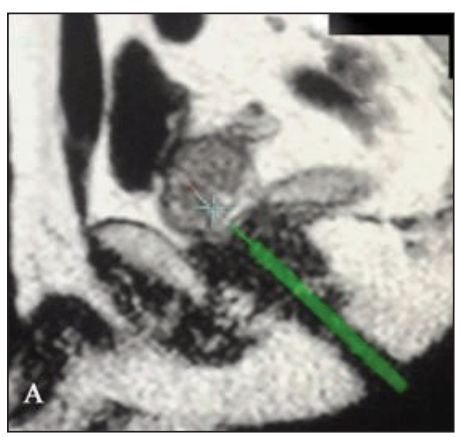

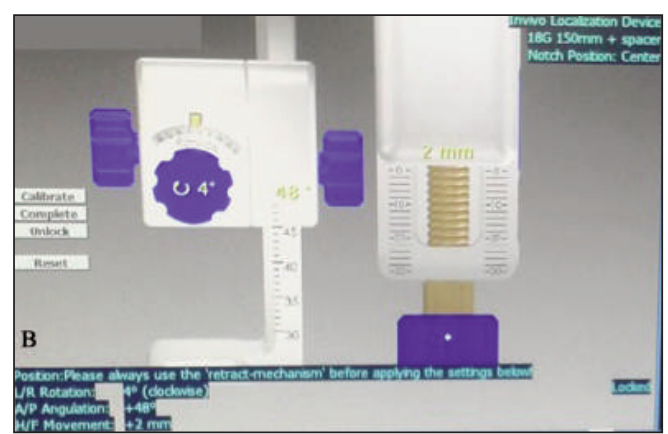


prone position on the $1.5 \mathrm{~T}$ MR scanner, using an 18-G automatic core-needle filled with Gadolinium, a non-magnetic portable biopsy device (DynaTRIM, Invivo, Gainesville, FL - Figure 1) and a dedicated software package for device tracking and target localization (DynaCAD, Invivo, Gainesville, FL). Oblique axial $\mathrm{T} 2 \mathrm{w}$ images were aligned with the needle guide in order to allow software registration showing three-dimensional adjustments required to align the track of the biopsy needle through the needle guide and the target lesion (Figure 1). After manual calibration adjustments on the arm of the biopsy device attached to the needle guide, sagittal T2w images in parallel with the long axis were obtained in order to confirm the correct position and the proper direction of the needle guide to the target; reconfirmation of the needle track was repeated until proper alignment was obtained. If targeting was not certain, due to lesion size or subjective judgment of the operator, subsequent axial and sagittal $\mathrm{T} 2 \mathrm{~W}$ images with the needle in place were obtained to detect needle position and be able to make adjustments for the next core (Figures 3a, 3b). A maximum of two biopsy cores were taken for each patient.

\section{Surgical procedure}

The HoLEP procedure was carried out by four experienced surgeons in either one of the two centers using the Lumenis ${ }^{\circledR}$ Versa Pulse ${ }^{\mathrm{TM}}$ Holmium laser delivering laser energy with a $550-\mu \mathrm{m}$ fiber set at $2.0 \mathrm{~J}$ and $60 \mathrm{~Hz}$ (maximum power of 120 W) and a 26Fr continuous-flow Storz laser resectoscope. A modified Gilling's technique (16) was employed and enucleated prostatic lobes were retrieved using Lumenis ${ }^{\circledR}$ VersaCut $^{\mathrm{TM}}$ Morcellator System. Continuous flow irrigation until next morning through a $20 \mathrm{~F}$ three-way catheter indwelled at the end of the surgery was placed. Catheter removal was executed at the second post-operative day in the event of no intercurred complication (e.g. hematuria, fever, acute urinary retention, etc.).

\section{Statistical analysis}

Overall patients $(\mathrm{n}=117)$ were dived in two groups, "IN-BORE MRI-GB" ( $\mathrm{n}=$ 57) and "mpMRI + TRUS-GB" $(\mathrm{n}=60)$ respectively based on the presence of a negative in-bore MRI-GB or a negative mpMRI with a contextual negative TRUS-GB prior to HoLEP.

Median values with interquartile ranges (IQR) and frequencies with proportions (\%) were reported for continuous and categorical variables respectively.

Differences between two groups were investigated with Mann-Whitney U test for continuous data, and chi-square test for categorical values.

Table 1. receptor inhibitor.
Univariate and multivariate logistic regression model was employed to investigate if any preoperative factors (Age, PSA, Prostate Volume, Adenoma Volume, Removed Tissue, PSA density > 15, negative in-bore MRI-GB) could be correlated with $\mathrm{iPCa}$ in patients who underwent HoLEP with a suspect of PCa.

IBM SPSS v.22 with a 2-sided significance level set at $\mathrm{P}<$ 0.05 , was used for statistical analysis.

\section{RESULTS}

A total amount of 117 patients [IN-BORE MRI-GB ( $\mathrm{n}=$ 57), mpMRI + TRUS-GB $(n=60)]$ were identified and included in the study. Preoperative clinical patients 'characteristics are listed in Table 1. Overall median age, PSA, prostate volume, adenoma volume and PSA density were

General and preoperative clinical characteristics.

\begin{tabular}{|c|c|c|c|c|}
\hline & $\begin{array}{c}\text { Overall } \\
(\mathrm{n}=117)\end{array}$ & IN-BORE MRI-GB & $\begin{array}{c}\text { mpMRI + TRUS-GB } \\
(\mathrm{n}=57)\end{array}$ & $\begin{array}{l}P \text { value } \\
(n=60\end{array}$ \\
\hline \multicolumn{5}{|l|}{ Age, years } \\
\hline Median (IQR) & $65.0(59.5-70.0)$ & $65.0(60.0-70.0)$ & $65.0(58.25-69.0)$ & 0.44 \\
\hline \multicolumn{5}{|l|}{$\mathrm{PSA}, \mathrm{ng} / \mathrm{ml}$} \\
\hline Median (IQR) & $6.20(5.40-9.90)$ & $5.96(5.26-10.0)$ & $6.00(4.82-9.87)$ & 0.66 \\
\hline DRE, $n(\%)$ & & & & 0.37 \\
\hline Negative & $95(81.2)$ & $44(77.2)$ & $51(85.0)$ & \\
\hline Positive & $22(18.8)$ & $13(22.8)$ & $9(15.0)$ & \\
\hline \multicolumn{5}{|l|}{ Prostate volume, cc } \\
\hline Median (IQR) & $80.0(60.0-101.0)$ & $68.0(58.0-97.0)$ & $84.0(70.0-115.0)$ & $0.01 *$ \\
\hline \multicolumn{5}{|l|}{ Adenoma volume, cc } \\
\hline Median (IQR) & $47.0(35.0-70.0)$ & $47(35.0-70.0)$ & $50.0(37.0-73.0)$ & 0.33 \\
\hline \multicolumn{5}{|l|}{ PSA Density, $n g / m l / c c$} \\
\hline Median (IOR) & $0.08(0.06-0.13)$ & $0.08(0.07-0.15)$ & $0.08(0.06-0.11)$ & 0,41 \\
\hline \multicolumn{2}{|c|}{ PSA Density > $0.15 \mathrm{ng} / \mathrm{ml} / \mathrm{cc}, \mathrm{n}(\%)$} & & & 0.06 \\
\hline № & $100(85.5)$ & $45(78.9)$ & $55(91.7)$ & \\
\hline Yes & $17(14.5)$ & $12(21.1)$ & $5(8.3)$ & \\
\hline \multicolumn{5}{|l|}{$\overline{Q_{m a x}} \mathrm{~mL} / \mathrm{sec}$} \\
\hline Median (IQR) & $9.6(6.8-9.5)$ & $11.0(10.2-12.0)$ & $8.7(8.0-11.0)$ & 0.11 \\
\hline \multicolumn{5}{|l|}{ IPSS score } \\
\hline Median (IQR) & $20.0(17.0-22.0)$ & $19.0(17.0-22.0)$ & $20.0(17.5-22.0)$ & 0.71 \\
\hline \multicolumn{5}{|l|}{ PRV, cC } \\
\hline Median (IQR) & $95(50-150)$ & $80(52-140)$ & $105(42-321)$ & 0.32 \\
\hline Drug assumption, $n(\%)$ & & & & 0.65 \\
\hline No & $15(12.2)$ & $6(10.5)$ & $9(15.0)$ & \\
\hline Alfa blocker & $43(36.8)$ & $24(42.1)$ & $19(31.7)$ & \\
\hline 5-ARI & $12(10.3)$ & $6(10.5)$ & $6(10.0)$ & \\
\hline 5-ARI + alfa-blocker & $47(40.2)$ & $21(36.8)$ & $26(43.7)$ & \\
\hline \multicolumn{2}{|c|}{ Previous acute urinary retention, $\mathrm{n}(\%)$} & & & 0.09 \\
\hline No & $105(89.7)$ & $54(94.7)$ & $51(85.0)$ & \\
\hline Yes & $12(10.3)$ & $3(5.3)$ & $9(15.0)$ & \\
\hline \multicolumn{2}{|l|}{ PIRADS v2 Score, n (\%) } & & & - \\
\hline$<3$ & $60(51.3)$ & $0(0.0)$ & $60(100.0)$ & \\
\hline 3 & $36(30.8)$ & $36(63.2)$ & $0(0.0)$ & \\
\hline 4 & $18(15.4)$ & $18(31.6)$ & $0(0.0)$ & \\
\hline 5 & $3(2.6)$ & $3(5.3)$ & $0(0.0)$ & \\
\hline \multicolumn{2}{|l|}{ Site Positive Area, n (\%) } & & & - \\
\hline Anterior & $48(84.2)$ & $48(84.2)$ & $0(0.0)$ & \\
\hline Posterior & $9(15.8)$ & $9(15.8)$ & $0(0.0)$ & \\
\hline \multicolumn{2}{|l|}{ Site Positive Area, n (\%) } & & & - \\
\hline Right lobe & $31(54.4)$ & $31(54.4)$ & $0(0.0)$ & \\
\hline Left lobe & $26(45.6)$ & $26(45.6)$ & $0(0.0)$ & \\
\hline
\end{tabular}

Continuous variables are shown as median (IQR) values while categorical as number (\%). Statistically significant values are considered as $p$ value $<0.05$.

PSA prostate specific antigen, DRE digital rectal examination, $Q_{\max }$ maximum peak urinary flow, IPSS international prostate symptoms score, QoL quality of life, PRV post-void volume, 5-ARI 5-aromatase

mpMRI multiparametric Magnetic Resonance Imaging. 
Table 2.

Perioperative surgical and histological outcomes.

\begin{tabular}{|c|c|c|c|c|}
\hline & $\begin{array}{c}\text { Overall } \\
(n=117)\end{array}$ & $\begin{array}{l}\text { IN-BORE MRI-GB } \\
\quad(n=57)\end{array}$ & $\begin{array}{c}\text { mpMRI + TRUS-GB } \\
(\mathrm{n}=60)\end{array}$ & $P$ value \\
\hline \multicolumn{5}{|c|}{ Surgery time (min) } \\
\hline Mean (SD) & $70.0(50.0-95.0)$ & $70.0(40.0-80.0)$ & $70.0(55.0-100.0)$ & 0.85 \\
\hline \multicolumn{5}{|c|}{ Removed tissue (gr) } \\
\hline Mean (SD) & $30.0(20.0-55.0)$ & $24.0(17.0-45.0)$ & $36.0(24.0-70.0)$ & 0.90 \\
\hline \multicolumn{5}{|c|}{ Catheterization time (days) } \\
\hline Median (IQR) & $2(2-2)$ & $2(2-2)$ & $2(2-2)$ & 0.32 \\
\hline \multicolumn{5}{|c|}{ Hospital stay (days) } \\
\hline Median (IQR) & $2(2-2)$ & $2(2-2)$ & $2(2-2)$ & 0.30 \\
\hline \multicolumn{5}{|c|}{ Peri-operative complications, $n(\%)$} \\
\hline No & $109(93.2)$ & $52(91.2)$ & $57(90.5)$ & \\
\hline Grade* 1 & $5(4.3)$ & $3(5.3)$ & $2(6.3)$ & \\
\hline Grade*2 & $3(2.5)$ & $2(3.5)$ & $1(3.2)$ & 0.36 \\
\hline \multicolumn{5}{|c|}{ Histopathology (\%) } \\
\hline Negative & $103(88.1)$ & $49(86.0)$ & $54(90.0)$ & \\
\hline $\mathrm{iPCa}$ & $14(12.0)$ & $8(14.0)$ & $6(10.0)$ & 0.50 \\
\hline \multicolumn{5}{|l|}{ pT Stage (\%) } \\
\hline pT1a & $10(71.4)$ & $6(75.0)$ & $4(66.7)$ & \\
\hline pT1b & $4(28.6)$ & $2(25.0)$ & $2(33.3)$ & 0.73 \\
\hline \multicolumn{5}{|c|}{ ISUP Grade Group (\%) } \\
\hline Group I & $11(78.6)$ & $6(75.0)$ & $5(83.3)$ & \\
\hline Group II & $3(21.4)$ & $2(25.0)$ & $1(16.7)$ & 0.71 \\
\hline
\end{tabular}

Continuous variables are shown as median (IQR) values while categorical as number (\%).

Statistically significant values are considered as $p$ value $<0.05$

pT stage pathologic T stage, ISUP International Society of Urological Pathology.

mpMRI multiparametric Magnetic Resonance Imaging.

Table 3.

Uni and multivariate logistic regression.

\begin{tabular}{|lcccc}
\hline \multirow{2}{*}{ Variables } & \multicolumn{2}{c}{ Univariate } & \multicolumn{2}{c|}{ Multivariate } \\
& $p$ value & OR $(95 \% \mathrm{Cl})$ & $\mathrm{p} \mathrm{value}$ & OR $(95 \% \mathrm{Cl})$ \\
\hline Age [continuous] (years) & $0.03^{*}$ & $1.12(1.01-1.25)$ & $0.02 *$ & $1.14(1.02-1.27)$ \\
\hline PSA [continuous] (ng/mL) & 0.15 & $1.09(0.96-1.23)$ & 0.08 & $1.12(0.99-1.28)$ \\
\hline Prostate Volume [continuous] (cc) & 0.41 & $1.01(0.99-1.02)$ & & \\
\hline Adenoma Volume [continuous] (cc) & 0.16 & $1.02(0.98-1.04)$ & & \\
\hline Removed Tissue [continuous] (gr) & 0.93 & $1.01(0.98-1.02)$ & & \\
\hline PSA density > 15 [yes vs no] (ng/mL/cc) & 0.44 & $1.73(0.42-6.99)$ & & \\
\hline Negative IN-BORE MRI-GB (yes vs no) & 0.50 & $1.46(0.47-4.53)$ & 0.37 & $1.72(0.51-5.77)$ \\
\hline
\end{tabular}

MRI-GB magnetic resonance imaging guided biopsy.

Statistically significant values are considered as $p$ value $<0.05$

$\mathrm{OR}$ odds ratio, $\mathrm{Cl}$ confidence interval, PSA prostate specific antigen.

65.0 years (IQR: $59.5-70.0$ ), $6.20 \mathrm{ng} / \mathrm{mL}$ (IQR: $5.40-$ 9.90), 80.0 cc (IQR: 60.0-101.0), 47.0 cc (IQR: 35.070.0) and $0.08 \mathrm{ng} / \mathrm{mL} / \mathrm{cc}$ (IQR: 0.06-0.13), respectively. A statistically significant difference was found between the two groups in terms of total prostate volume with $68 \mathrm{cc}$ (IQR: 58.0-97.0) in the IN-BORE MRI-GB group and 84 cc (IQR: 70.0-115.0) in the mpMRI + TRU-GB group ( $\mathrm{p}=$ $0.01)$. However no statistically differences were found between the two groups in terms of adenoma volume (47 cc IQR: 35.0-70.0 versus 50 cc IQR: 37.0-73.0; $\mathrm{p}=0.33$ ) and the other clinical preoperative characteristics.

Patients in both groups presented moderate to severe lower tract urinary symptoms with affected quality of life, based on the IPSS, and a bladder outflow obstruction with decreased peak urinary flow $\left(\mathrm{Q}_{\max }\right)$. Median IPSS was 19 (IQR: 17.0-22.0) in the IN-BORE MRI-GB group and 20 (IQR: 17.5-22.0) in the mpMRI + TRUSGB $(\mathrm{p}=0.71)$.

No statistically differences were recorded between the two groups in preoperative drug assumption and previous

\section{Discussion}

acute urinary retention.

The majority of patients with a negative IN-BORE MRI-GB presented either a posterior $(84.2 \%)$ or a a PI-RADS-v2 Score 3 area $(63.2 \%)$.

Perioperative surgical outcomes, as reported in Table 2, were found to be comparable in terms of surgery time, removed issue, catheterization time, hospital stay and perioperative complication. No statistically different rates $(p=0.50)$ of iPCa detected in the resected tissue of IN-BORE MRI-GB group (14\%) in comparison with mpMRI + TRUS-GB group (10\%) were shown at final pathology examination.

Comparable pT stage and ISUP Grade Group in iPCa stratification were found with pTla stage and ISUP Grade Group I (Gleason Score 3+3) 75.0\% versus $66.7 \%$ $(\mathrm{p}=0.73)$ and $75.0 \%$ versus $63.3 \%(\mathrm{p}=$ 0.71 ) respectively in IN-BORE MRI-GB and mpMRI + TRUS-GB group.

Univariate analysis (Table 3) showed that only Age (OR 1.12; 95\% CI: 1.01-1.25; $\mathrm{p}=0.03)$ was correlated with iPCa after HoLEP, whereas a preoperative negative in-bore MRI-GB wasn't statistically related to iPCa (OR 1.46; 95\% CI: 0.47-4.53; p = $0.50)$ such as PSA Density > 0.15 $n g / m L / c c(p=0.44)$, PSA $(p=0.15)$, prostate volume $(p=0.41)$, adenoma volume $(p=0.16)$, removed tissue $(p=$ 0.93). In a multivariate predictive model a statistically significant correlation with Age as an independent predictive factor of iPCa was also found (OR 1.14; 95\% CI: 1.02-1.27; $\mathrm{p}=0.02$ ) while no correlations were revealed with PSA (OR 1.12; 95\% CI: 0.99-1.28; $p=0.08$ ) and a negative inbore MRI-GB (OR 1.72; 95\% CI: 0.515.77; $\mathrm{p}=0.37$ ) (Table 3$)$.

HoLEP represents a modern less-invasive treatment of symptomatic BPE with demonstrated safety and effectiveness with long terms results, even in a randomized study (17). Respect to other laser BPE surgery, HoLEP leads to a transurethral enucleation which sometimes the retrieval of an iPCa in the final pathology. Elkoushy et al. (18) conducted a prospective study demonstrating that active surveillance $(19,20)$ might be a safe clinical option in managing iPCa diagnosis after HoLEP, especially because radiotherapy or radical prostatectomy, even if necessary sometimes, often negatively affect quality of life (21). Therefore, a different novel clinical approach is necessary when a PCa suspicion is present before to schedule surgery for BPE.

Purpose of our study was to evaluate the role of a negative in-bore MRI-GB in comparison to a negative mpMRI and a contextual negative transrectal ultrasound guided 
biopsy of the prostate, before an HoLEP surgery, with regard to incidental prostate cancer findings in individuals with a preoperative suspicion of prostate cancer. All patients of our retrospective multicentric study were symptomatic due BOO secondary to BPE, had a raised serum PSA and/or an abnormal DRE, underwent to a pre-operative mpMRI and PCa was excluded either with a negative in-bore magnetic resonance imaging guided prostate biopsy (IN-BORE MRI-GB group) or an additional standard prostate biopsy to the negative mpMRI (mpMRI + TRUS-GB group).

Both study groups presented pre-surgery assessments e peri-operative surgical outcomes with no statistically significant differences, besides total prostate volume but not adenoma volume, demonstrating low rates of complications, short hospital stay (median 2 days; IQR 2-2) and catheterization time (median 2 days; IQR 2-2).

In the overall selected population of our study, final pathology examination showed a rate of iPCa (12\%) which is comparable to the interval available in the present literature (8.1-15\%) (22-24).

The explanation to this range might be found in the various baseline characteristics of the patients, which usually are due to merging individuals with normal PSA and DRE to patients with suspicion of PCa.

In fact Herlemann et al. (24), in a sub-analysis of their HoLEP study arm, found a 40\% iPCa rate in a sub-cohort of patients with a preoperative negative TRUS-GB, highlighting the need of a different preoperative diagnostic approach when a suspect of $\mathrm{PCa}$ is present before $\mathrm{BPE}$ surgery.

Several preoperative parameters, such as older age, preoperative PSA, smaller prostate volume, preoperative TRUS-GB, were pointed as possible predictor of iPCa before BPE surgery. Bhojani et al. (23) in their study demonstrated with their regression model that age prior surgery is an independent predictive factor for $\mathrm{iPCa}$ before HoLEP.

In our experience mpMRI proved to be a valuable preoperative tool not only, as demonstrated,in planning a precise and safe nerve sparing in patients scheduled for radical prostatectomy (25), but also to exclude $\mathrm{PCa}$ before HoLEP either with a negative finding or using the same MRI in order to guide a precise biopsy in a suspicious area.

Our reported rate of $12 \%$ of iPCa after HoLEP in patients with a suspect preoperative $\mathrm{PCa}$ is lower both than the $40 \%$ of the sub cohort of Herlemann et al. (24) and the recent $23.1 \%$ of 359 patients treated with HoLEP and TRUS-GB recently reported by Kim et al. (26).

In our study a negative in-bore MRI-GB showed a good reliability in order to safely exclude a PCa and didn't show any statistically difference in percentage of iPCa compared to a negative mpMRI with a contextual TRUSGB, $14 \%$ versus $10 \%(\mathrm{p}=0.50)$, and in $\mathrm{pT}$ stage and ISUP Grade Group distribution ( $\mathrm{p}=0.73$ and $\mathrm{p}=0.71$ respectively). Both in univariate (OR 1.46; 95\% CI: 0.47 4.53; $\mathrm{p}=0.50$ ) and multivariate (OR $1.72 ; 95 \% \mathrm{CI}$ : $0.51-5.77 ; \mathrm{p}=0.37$ ) regression analysis model a negative in-bore MRI-GB wasn't and independent predictive factor of iPCa before HoLEP while at the same regression model age was found to significant either in univariate
(OR 1.12; 95\% CI: 1.01-1.25; $\mathrm{p}=0.03$ ) and multivariate (OR 1.14; 95\% CI: 1.02-1.27; $\mathrm{p}=0.02$ ).

The retrospective nature of our study and the absence of a randomization are the main limitations and secondly the two groups were not matched. Patients' data with positive in-bore MRI-GB or TRUS-GB were not available thus a prospective randomized data collection is needed in order to confirm our preliminary results.

Moreover, our data didn't let a stratification based on an $80 \mathrm{cc}$ cut-off prostate volume, and lastly there is both a lack of a long-term oncologic follow-up and the comparison with the histologic gold standard for prostate cancer diagnosis (radical prostatectomy specimen) since the presence of cancer risk in the residual peripheral prostate.

Despite these important limitations, our study represents a selected cohort of patients due the inclusion of only individuals with serum PSA $>4 \mathrm{ng} / \mathrm{mL}$ and/or abnormal DRE and our data represent one of the first studies available in the clinical use of a prostate mpMRI and an inbore MRI-GB before HoLEP. However, in order to deeply investigate and confirm our preliminary results randomized trials and further investigations are needed.

\section{Conclusions}

Our results show that a negative preoperative in-bore MRI guided prostate biopsy before HoLEP in patients with raised serum PSA and/or abnormal DRE leads to low rate of iPCa. Therefore, including a mpMRI and an eventual in-bore MRI-GB in a novel clinical evaluation might avoid unnecessary standard TRUS-GB biopsies and represents a novel clinical approach in patients eligible to HoLEP due symptomatic BOO secondary to BPE who presents a suspicion of PCa.

\section{References}

1. De Ridder D, Roumeguère T, Kaufman L. Urgency and other lower urinary tract symptoms in men aged $\geq 40$ years: a Belgian epidemiological survey using the ICIQ-MLUTS questionnaire. Int J Clin Pract. 2015; 69:358-65.

2. Noale M, Maggi S, Artibani W, et al. Pros-IT CNR: an Italian prostate cancer monitoring project. Aging Clin Exp Res. 2017; 29:165-72

3. Vagnoni V, Bianchi L, Borghesi M, et al. Adverse features and competing risk mortality in patients with high-risk prostate cancer. Clin Genitourin Cancer. 2017; 15:e239-48.

4. Schiavina R, Bianchi L, Borghesi M, et al. Predicting survival in node-positive prostate cancer after open, laparoscopic or robotic radical prostatectomy: A competing risk analysis of a multi-institutional database. Int J Urol. 2016; 23:1000-8.

5. Mottet N, Bellmunt J, Bolla M, et al. EAU-ESTRO-SIOG Guidelines on prostate cancer. Part 1: Screening, diagnosis, and local treatment with curative intent. Eur Urol. 2017; 71:618-29.

6. Jones JS. Saturation biopsy for detecting and characterizing prostate cancer. BJU Int. 2007; 99:1340-4.

7. Serefoglu EC, Altinova S, Ugras NS, et al. How reliable is 12-core prostate biopsy procedure in the detection of prostate cancer? Can Urol Assoc J 2013; 7:E293-298.

8. Manfredi M, Mele F, Garrou D, et al. Multiparametric prostate 
MRI: technical conduct, standardized report and clinical use. Minerva Urol Nefrol. 2018; 70:9-21.

9. Vagnoni V, Brunocilla E, Bianchi L, et al. State of the art of PET/CT with 11-choline and 18F-fluorocholine in the diagnosis and follow-up of localized and locally advanced prostate cancer. Arch Esp Urol. 2015; 68:354-70.

10. Porpiglia F, Manfredi M, Mele F, et al. Diagnostic pathway with multiparametric magnetic resonance imaging versus standard pathway: results from a randomized prospective study in biopsy-naïve patients with suspected prostate cancer. Eur Urol. 2017; 72:282-8.

11. Schoots IG, Roobol MJ, Nieboer D, et al. Magnetic resonance imaging-targeted biopsy may enhance the diagnostic accuracy of significant prostate cancer detection compared to standard transrectal ultrasound-guided biopsy: a systematic review and meta-analysis. Eur Urol. 2015; 68:438-50.

12. Schiavina R, Vagnoni V, D’Agostino D, et al. "In-bore" MRIguided prostate biopsy using an endorectal nonmagnetic device: a prospective study of 70 consecutive patients. Clin Genitourin Cancer. 2017; 15:417-27.

13. Krambeck AE, Handa SE, Lingeman JE. Experience with more than 1,000 holmium laser prostate enucleations for benign prostatic hyperplasia. J Urol. 2013; 189(1 Suppl):S141-145.

14. Naspro R, Freschi M, Salonia A, et al. Holmium laser enucleation versus transurethral resection of the prostate. Are histological findings comparable? J Urol. 2004; 171:1203-6.

15. Weinreb JC, Barentsz JO, Choyke PL, et al. PI-RADS Prostate Imaging - Reporting and Data System: 2015, Version 2. Eur Urol. 2016; 69:16-40.

16. Gilling PJ, Kennett K, Das AK, et al. Holmium laser enucleation of the prostate (HoLEP) combined with transurethral tissue morcellation: an update on the early clinical experience. J Endourol. 1998; 12:457-9.

17. Lourenco T, Pickard R, Vale L, et al. Alternative approaches to endoscopic ablation for benign enlargement of the prostate: systematic review of randomised controlled trials. BMJ. 2008; 337:a449.
18. Elkoushy MA, Elshal AM, Elhilali MM. Incidental prostate cancer diagnosis during holmium laser enucleation: assessment of predictors, survival, and disease progression. Urology. 2015; 86:552-7.

19. Schiavina R, Borghesi M, Brunocilla E, et al. The biopsy Gleason score $3+4$ in a single core does not necessarily reflect an unfavourable pathological disease after radical prostatectomy in comparison with biopsy Gleason score 3+3: looking for larger selection criteria for active surveillance candidates. Prostate Cancer Prostatic Dis. 2015; 18:270-5.

20. Grasso AA, Cozzi G, DE Lorenzis E, et al. Multicenter analysis of pathological outcomes of patients eligible for active surveillance according to PRIAS criteria. Minerva Urol Nefrol. 2016; 68:237-41.

21. Porreca A, Noale M, Artibani W, et al. Disease-specific and general health-related quality of life in newly diagnosed prostate cancer patients: the Pros-IT CNR study. Health Qual Life Outcomes. 2018; 16:122.

22. Nunez R, Hurd KJ, Noble BN, et al. Incidental prostate cancer revisited: Early outcomes after holmium laser enucleation of the prostate. Intern J Urol. 2011; 18:543-7.

23. Bhojani N, Boris RS, Monn MF, et al. Coexisting prostate cancer found at the time of holmium laser enucleation of the prostate for benign prostatic hyperplasia: predicting its presence and grade in analyzed tissue. J Endourol. 2015; 29:41-6.

24. Herlemann A, Wegner K, Roosen A, et al. "Finding the needle in a haystack": oncologic evaluation of patients treated for LUTS with holmium laser enucleation of the prostate (HoLEP) versus transurethral resection of the prostate (TURP). World J Urol. 2017; 35:1777-82.

25. Schiavina R, Bianchi L, Borghesi M, et al. MRI Displays the prostatic cancer anatomy and improves the bundles management before robot-assisted radical prostatectomy. J Endourol. 2018; 32:315-21.

26. Kim KH, Kim SW, Son HS, et al. Role of Holmium laser enucleation of the prostate to increase cancer detection rate in patients with gray-zone PSA level. Minerva Urol Nefrol. 2019; 71:72-8.

\section{Correspondence}

Porreca Angelo, MD

angeloporreca@gmail.com

D'Agostino Daniele, MD

daniele.dagostino@casacura.it

Corsi Paolo, MD

paolo.corsi@casacura.it

Romagnoli Daniele, MD

danieleromagnoli87@gmail.com

Del Rosso Alessandro, MD

adelrosso@casacura.it

Artibani Walter, MD

prof.artibani@gmail.com

Giampaoli Marco, MD (Corresponding Author)

marco.giampaoli@casacura.it

Robotic Urology and Mini Invasive Urologic Surgery Unit, Abano Terme Hospital

Piazza Cristoforo Colombo, 1, 35031 Abano Terme (Padova) (Italy)

Vigo Mario, MD

Department of Radiology, Abano Terme Hospital, Padua, Italy

Schiavina Riccardo, MD

rschiavina@yahoo.it

Brunocilla Eugenio, MD

eugenio.brunocilla@unibo.it

Department of Specialistic, Experimental and Diagnostic Medicine, Urology,

Alma Mater Studiorum-University of Bologna, S. Orsola Hospital

Via Pelagio Palagi, 9, 40138 Bologna (Italy) 